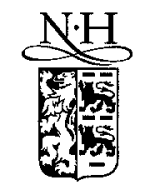

\title{
Energy product for nanostructured two-phase magnets
}

\author{
Ching-Ming Lee, Ching-Ray Chang * \\ Department of Physics, National Taiwan University, Taipei, Taiwan, ROC
}

\begin{abstract}
We have calculated the influence of volume fraction of the soft phase in a nanostructured two-phase magnet on its energy product. The result shows that the energy product depends not only on the size of the soft inclusion but also on its volume fraction.
\end{abstract}

Keywords: Energy product; Two-phase magnets

It is well known that both the sizes of the hard and the soft phase play key roles in the two-phase magnets. The effect of small grain size of the hard phase leads to exchange coupling between these grains and thus enhances the remanence $[1,2]$. On the other hand, exchange interactions between different phases preserve a high coercivity for the small size of the soft phase [3,4]. Some experimental works [2,5] and numerical result [3] show that the volume fraction of the soft phase also has influence on energy product. Here we propose an analytical model to investigate this problem.

For simplicity, we consider the oriented two-phase magnet as an infinite array of rectangular two-phase prisms. The soft-phase prisms that have cross section $D^{2}$ and height $t$ are situated within a three-dimensional lattice of hard-phase material. Here we use the Fourier method [6] to calculate the interaction field of prisms. Using the principle of superpositions the interaction field at the center of the soft-phase prism $H_{i}$ can be written as $H_{i}=H_{t}-H_{p}$, where $H_{t}$ is the field produced by periodically arranged prisms with magnetization $M_{s}-M_{h}$ and $H_{p}$ is the field due to a single soft-phase prism which should be subtracted. Assuming the gap between prisms is $g$ we can write down $H_{t}$ and $H_{p}$ analytically [6],

$$
\begin{aligned}
& \frac{H_{t}}{4 \pi\left(M_{s}-M_{h}\right)}=-\frac{4 D}{(D+g) \pi} \sum_{n=1}^{\infty} \frac{1}{n} \sin \frac{k_{n} D}{2} F\left(k_{n}, t, g\right) \\
& -\frac{4}{\pi^{2}} \sum_{m, n 1}^{\infty} \frac{1}{m n} \sin \frac{k_{n} D}{2} \sin \frac{k_{m} D}{2} F\left(k_{n m}, t, g\right) \\
& -\frac{D^{2}}{(D+g)^{2}}
\end{aligned}
$$

\footnotetext{
${ }^{*}$ Corresponding author. Email: crchang6@phy.ntu.edu.tw.
}

$$
\frac{H_{p}}{4 \pi M_{s}}=-\frac{2}{\pi} \tan ^{-1}\left[\frac{D^{2}}{t \sqrt{2 D^{2}+t^{2}}}\right],
$$

where

$$
\begin{aligned}
& F(k, t, g)=\exp \left(-\frac{k t}{2}\right)\left[\frac{1-\exp (-k g)}{1-\exp (-k(t+g))}\right], \\
& k_{j}=(2 j \pi) /(D+g), j=n, m,
\end{aligned}
$$

and

$k_{n m}=\sqrt{k_{n}^{2}+k_{m}^{2}}$.

With the information of local fields, the calculation of the nucleation field of the two-phase magnets can thus be simplified by a model which a single soft inclusion was embedded in an infinite hard matrix. For simplicity, we use a cylindrical grain instead of a rectangular prism. Moreover, it is plausible to choose the curling mode as the mode of magnetization reversal to reduce the demagnetization energy. Hence, the total magnetic energy of such a composition can be written as

$$
\begin{aligned}
E= & 2 \pi \int_{0}^{R} r \mathrm{~d} r\left[G\left(A_{s}, K_{s}, M_{s}, \theta_{1}\right)+E_{D}\right] \\
& +2 \pi \int_{R}^{\infty} r \mathrm{~d} r G\left(A_{h}, K_{h}, M_{h}, \theta_{2}\right)
\end{aligned}
$$

with

$$
\begin{aligned}
G(A, K, M, \theta)= & A\left[\left(\frac{\mathrm{d} \theta}{\mathrm{d} r}\right)^{2}+\frac{1}{r^{2}} \sin ^{2} \theta\right] \\
& +K \sin ^{2} \theta-M H \cos \theta
\end{aligned}
$$

and

$E_{D}=\frac{1}{2}\left(M_{s}^{2}-M_{h}^{2}\right) N \cos ^{2} \theta_{1}$, 
where $G$ represents the sum of exchange, anisotropy, Zeeman energy densities and $E_{D}$ is the demagnetization energy density in which $N$ is the demagnetization factor of the cylindrical grain. $A_{j}, K_{j}$ and $M_{j}(j=h, s)$ are the exchange constants, uniaxial crystalline anisotropy constants, and magnetizations of the hard and soft regions. $H$ is the sum of the external applied field $H_{a}$ and the local interaction field $H_{i}$. The angles $\theta_{j}(j=1,2)$ of the magnetizations are taken with respect to the easy axis. When nucleation occurs, the magnetization has a small deviation from the original uniform state and thus can be linearized. Using the variation method to minimize the linearized energy function we obtain the characteristic equation in which the nucleation field satisfies:

$\mu_{1} \frac{J_{0}\left(\mu_{1}\right)}{J_{1}\left(\mu_{2}\right)}+p \mu_{2} \frac{K_{0}\left(\mu_{2}\right)}{K_{1}\left(\mu_{1}\right)}-1+p=0$.

Here we use the reduced units as follows:

$\rho=r / R, \quad R_{s}=\sqrt{A_{s}} / M_{s}, \quad S=R / R_{s}$,

$Q_{s}=K_{s} / 2 \pi M_{s}^{2}, \quad Q_{h}=K_{h} / 2 \pi M_{h}^{2}$,

$p=A_{h} / A_{s}, \quad q=M_{h} / M_{s}, \quad h=H / 4 \pi M_{s}$

and

$\mu_{1}=\sqrt{2 \pi S^{2}\left[-h-Q_{s}+\frac{N}{4 \pi}\left(1-q^{2}\right)\right]}$,

$\mu_{2}=\sqrt{2 \pi S^{2} \frac{q}{p}\left(q Q_{h}+h\right)}$.

$J_{1}, J_{0}$ and $K_{1}, K_{0}$ are the first type of Bessel and modified Bessel functions of order 1 and 0 , respectively.

Fig. 1 shows the relations between the nucleation field and the size of the soft grain with different volume fraction

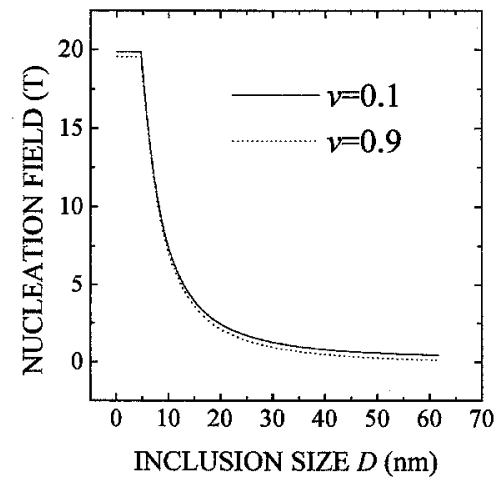

Fig. 1. Nucleation field as a function of inclusion size $D$ for volume fraction of soft phase $v=0.1$ and 0.9 . We use data for the $\mathrm{Sm}_{2} \mathrm{Fe}_{17} \mathrm{~N}_{3} /(\alpha$-Fe) system (see Ref. [1]).

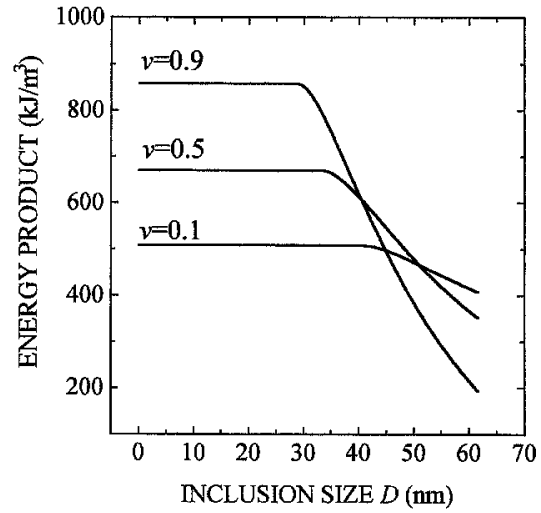

Fig. 2. Energy products of different volume fractions of soft phase all have plateau in the small grain size region and then decay with increasing grain size. The values used here are the same as Fig. 1.

$v$. Here we only consider the case of spherical-like shape, i.e., $t=D$. We can see that, as expected [6], a higher volume fraction causes a lower nucleation field. In the calculation of remanence, to estimate the theoretical upper limit; we assume the hysteresis is a square loop, hence the remanence is only the volume average of the saturation magnetizations of both phases. It would be smaller in the realistic isotropic cases [2,3]. Therefore, owing to the large saturation magnetization of the soft phase and enhancement of remanence, a high energy product with acceptable coercivity can be expected. In fact, from Fig. 2 we can see this is true only when the size of the soft inclusion is small enough.

In summary, with a high volume fraction we can increase the energy product in the case of small, soft inclusions and reduce the price because of low consumption of rare earth. Therefore, the volume fraction of the soft phase plays a key role in two-phase magnet.

\section{References}

[1] H. Fukunaga and H. Inoue, Jpn. J. Appl. Phys. 31 (1993) 1347.

[2] A. Manaf, R.A. Buckley and H.A. Davies, J. Magn. Magn. Mater. 128 (1993) 302.

[3] T. Schrefl, R. Fischer and H. Kronmüller, J. Appl. Phys. 76 (1994) 7053.

[4] R. Skomski and J.M.D. Coey, Phys. Rev. B 48 (1993) 15812.

[5] M.N.K. Willcox, J.M. Williams, M. Leonowicz, A. Manaf and H.A. Davies, Proc. 13th Intl. Workshop on Rare Earth Magnets, eds. Mainwaring et al. (Birmingham Univ., 1994) p. 443.

[6] Ching-Ray Chang, PA1-7, Compumag \#5, Berlin, Germany (1995). 